\title{
Harvested Energy and Spectral Efficiency Trade-offs in Multicell MIMO Wireless Networks
}

\author{
Tien Ngoc HA, Ha Hoang KHA \\ Faculty of Electrical and Electronics Engineering, Ho Chi Minh City University of Technology, VNU-HCM, Vietnam \\ hntien.hcmut@gmail.com,hhkha@hcmut.edu.vn \\ Submitted September 9, 2018 / Accepted January 20, 2019
}

\begin{abstract}
The paper focuses on designing precoding matrices in multi-cell multiple-input multiple-output (MIMO) simultaneous wireless information and power transfer networks (SWIPT) where the sets of users are selected for data transmission in each time slot and the unselected users are dedicated to energy harvesting. The precoding design for the SWIPT problem is formulated as a general multi-objective maximization problem, in which the sum-rate (SR) and sum harvested energy (SHE) are maximized simultaneously under the transmit power constraints. Since the objective function of the maximization problem is not concave in the design matrix variables, it is difficult to directly obtain the optimal solutions. To tackle this challenge, we recast the SR function into one more amenable by applying the connection between the minimum mean square error and achievable data rate. In addition, to deal with the non-concavity of the harvested energy function, we derive its concave minorant. Then, we develop an efficient iterative algorithm based on alternating optimization (AO) to obtain the optimal precoders. We also analyze the convergence and computational complexity of the proposed algorithm. Finally, by numerical simulation results we investigate the trade-offs between the SR and SHE.
\end{abstract}

\section{Keywords}

Multicell MU-MIMO, SWIPT, spectral efficiency, precoding design

\section{Introduction}

In the last decade, the explosively growing demands for high data rate and ubiquitous wireless communication applications have triggered an urgent need to improve the spectral efficiency (SE) in the next generation of wireless communication networks. The fifth generation $(5 \mathrm{G})$ wireless network is expected to provide ultra high data rates to meet the demands of high data rate applications and the massive number of wireless connected devices [1]. Thus, the SE performance metric is of importance for the wireless communication system designs. The authors of [2] optimized the SE by designing the precoders using particle swarm optimiza- tion for single-cell multiple-input multiple-output (MIMO) broadcast channels. The coordinated multiple small cell systems with multi-antenna base stations (BSs) in which the BSs jointly design the transmit precoders to manage interuser interference are known as a efficient means to improve the SE [3], [4]. In [3], the authors designed the precoders to maximize the sum-rate or minimum user rate by successive convex quadratic program in full-duplex MIMO multicell networks. With the significantly increasing number of wireless connected devices and dense deployment of multiple small cells in the next generation of wireless networks, the power consumption has recently received special attention due to its economic and ecological concerns [5]. Reference [6] investigated the energy efficiency for cognitive radio networks.

Towards to green wireless communications, energy harvesting $(\mathrm{EH})$ techniques which power-constrained wireless devices can harvest energy from the received radio frequency (RF) signals to prolong the operation time have recently received considerable attention [7-11]. The EH techniques have been studied on various aspects, for example, beamforming/precoding designs [8-11] and the rate and energy trade-off characteristics [12]. The EH problems have been also investigated in different system models: cognitive ratio networks [13], multi-user multiple-input multiple-output (MU-MIMO) interference channels [14], [15] and physical layer security [16], [17]. In this paper, we are concerned with multicell MU-MIMO simultaneous wireless information and power transfer network (SWIPT) wireless networks in which the sets of users are selected to receive the signals from downlink transmission while the idle users are able to harvest energy from the RF signals of the downlink channel signals.

Related works: The authors of [18] proposed an opportunistic interference alignment (OIA) for uplink MIMO wireless networks in which the groups of users are selected for uplink transmission while the unselected users harvest energy from the RF signals transmitted by the selected users. The authors developed an intra-cluster performance awareness and OIA schemes to balance the system sum rate (SR) and sum harvested energy (SHE). In [19], the authors considered the multicell multiuser SWIPT system in which each user 
equipment (UE) is equipped with a single antenna. Each UE can decode information and harvest energy simultaneously by using a power splitting technique. By scheduling a single UE for each time slot, the authors investigated the tradeoffs between the rate and energy of the system. Concerning user selection strategies in multicell networks with multiple single-antenna users, the authors in [26] proposed efficient user selection schemes based on the pre-defined structures of the precoding matrices (namely, distributed zero-forcing or distributed virtual SINR) to potentially maximize the system sum-rate. Alternatively, the authors in [20] proposed a multiobjective optimization approach to maximize the data rates and harvested energy in a single cell multi-user broadcast system. More generally, reference [22] designed the precoding matrices for either SR maximization under the quality of service (QoS) and energy harvesting constraints or harvested energy maximization under throughput constraints in the full-duplex multicell MU-MIMO wireless networks.

Contributions: Different from the above mentioned works, in this paper, we consider the downlink multicell MU-MIMO models and we aim at simultaneously maximizing the network SR and SHE by a means of multi-objective optimization. Our major contributions are to develop an efficient iterative algorithm to investigate the SE and harvested energy trade-offs for the downlink multi-cell MU-MIMO SWIPT networks. To efficiently handle intra-cell and intercell interference and to improve the SE, we propose a user scheduling scheme in which each BS exploits the channel state information (CSI) to select a set of users for data transmission and, then, the idle users which are not selected for information transmission harvest energy to prolong their battery lifetime. We first formulate the precoding design as an optimization problem in which the SR and SHE are simultaneously maximized subject to transmit power constraints at the BSs. The formulated problem of maximizing the weighted sum of SR and SHE is a nonconvex optimization one and, thus, it is mathematically challenging to obtain the analytical optimal solutions. To tackle with the nonconcavity of the SR function, we exploit the relationship between the SR maximization and mean squared error (MSE) minimization [23]. In addition, to handle the non-concavity of the harvested energy function, we derive its global lower bound by exploiting the convexity of the harvested energy function [22], [24]. As a result, we propose a numerical iterative algorithm based on alternating optimization $(\mathrm{AO})$ to obtain the optimal precoders for the objective maximization. We also provide the convergence and computational complexity analysis of the proposed method. By numerical simulations, we study the convergence of the proposed method and investigate the trade-off between the SE and harvested energy in multicell SWIPT wireless networks. It is important to remark that the multi-objective optimization which is similar to our approach was investigated in [20]. However, there are several key differences between the work in [20] and our study in the present paper. First, reference [20] studied the singlecell model while our work focuses on the multicell system model. Different from the single cell scenario in which there exists only (intra-cell) inter-user interference, the multicell models exhibit the additional inter-cell interference. Thus, the interference management in multicell systems is more complicated than that in single cell models. As a result, the precoding designs to suppress interference and to maximize the sum-rate in multicell systems are more involved and more challenging due to the nonlinearly decoupling of the precoding strategies of base stations in cells. Second, reference [20] focused on designing the transmit covariance matrices by using the majorization-minimization method to design the transmit covariance matrices. In contrast, our paper focuses on directly designing of precoding matrices by the alternating optimization algorithm.

The remainder of the paper is organized as follows. In Sec. 2, we introduce the signal and system models of multicell MU-MIMO SWIPT networks and, then, formulate the precoding design as an optimization problem. In Sec. 3, we derive the iterative algorithm to obtain the optimal precoders. Section 4 provides numerical simulation results. Finally, the conclusions are given in Sec. 5.

Notations: Matrices (vectors) are represented by boldface upper (lower) case letters. The Hermitian transposition, trace and determinant of matrix $\mathbf{X}$ are denoted by $\mathbf{X}^{\mathrm{H}},\langle\mathbf{X}\rangle$, and $|\mathbf{X}|$, respectively. $\mathbf{I}$ is an identity matrix with an appropriate dimension. $\mathbb{E}($.$) represents an expectation operation.$ A complex Gaussian random vector $\mathbf{x}$ with mean $\overline{\mathbf{x}}$ and covariance $\mathbf{R}_{\mathbf{z}}$ is denoted by $\mathbf{x} \sim \mathcal{C N}\left(\overline{\mathbf{x}}, \mathbf{R}_{\mathbf{x}}\right)$.

\section{System Model and Problem Formulation}

We consider a downlink transmission system of a cluster of $L$ cells as depicted in Fig. 1 in which the BS in cell $\ell$, denoted by $\mathrm{BS}_{\ell}, \ell \in \mathcal{L}=\{1, \ldots, \mathrm{L}\}$ is equipped $M_{\ell}$ transmit antennas. The number of users in cell $\ell$ is $K_{\ell}$. UE $k$ in cell $\ell$ referred to as $\mathrm{UE}_{\ell, k}, k \in \mathcal{K}_{\ell}=\left\{1, \ldots, K_{\ell}\right\}$ is equipped $N_{\ell, k}$ antennas. For each time slot, $\mathrm{BS} \mathrm{S}_{\ell}$ can support $S_{\ell}$ users $\left(S_{\ell} \leq K_{\ell}\right)$ in its coverage for data transmission. To prolong the battery lifetime, the idle users can harvest energy from RF signals. The sets of selected and unselected users in cell $\ell$ for each time slot are denoted by $\Pi_{\ell}=\left\{\pi_{\ell}(1), \ldots, \pi_{\ell}\left(S_{\ell}\right)\right\}$ and $\Phi_{\ell}=\left\{\phi_{\ell}(1), \ldots, \phi_{\ell}\left(K_{\ell}-S_{\ell}\right)\right\}$, respectively. It is assumed that $\Pi_{\ell} \cap \Phi_{\ell}=\varnothing$ and $\Pi_{\ell} \cup \Phi_{\ell}=\mathcal{K}_{\ell}$, which means that each user operates either as an information decoding receiver (IDR) or as an $\mathrm{EH}$ receiver (EHR).

Let $\mathbf{s}_{\ell, k} \in \mathbb{C}^{d_{\ell, k} \times 1}$ be $d_{\ell, k}$ independent data streams which are transmitted from $\mathrm{BS}_{\ell}$ to $\mathrm{UE}_{\ell, k}$. Without loss of generality, we assume that $\mathbb{E}\left(\mathbf{s}_{\ell, k} \mathbf{s}_{\ell, k}^{\mathrm{H}}\right)=\mathbf{I}_{d_{\ell, k}}$ and $\mathbb{E}\left(\mathbf{s}_{\ell, k} \mathbf{s}_{i, j}^{\mathrm{H}}\right)=\mathbf{0}$ for $(\ell, k) \neq(i, j)$. BS $\mathrm{B}_{\ell}$ uses the precoding matrix $\mathbf{V}_{\ell, k} \in \mathbb{C}^{M_{\ell} \times d_{\ell, k}}$ to linearly process the data streams of $\mathrm{UE}_{\ell, k}$. For compact notation, we denote the set of all precoding matrices by $\mathbf{V}=\left\{\mathbf{V}_{\ell, k}\right\}_{\ell \in \mathcal{L}, k \in \mathcal{K}_{\ell}}$. 


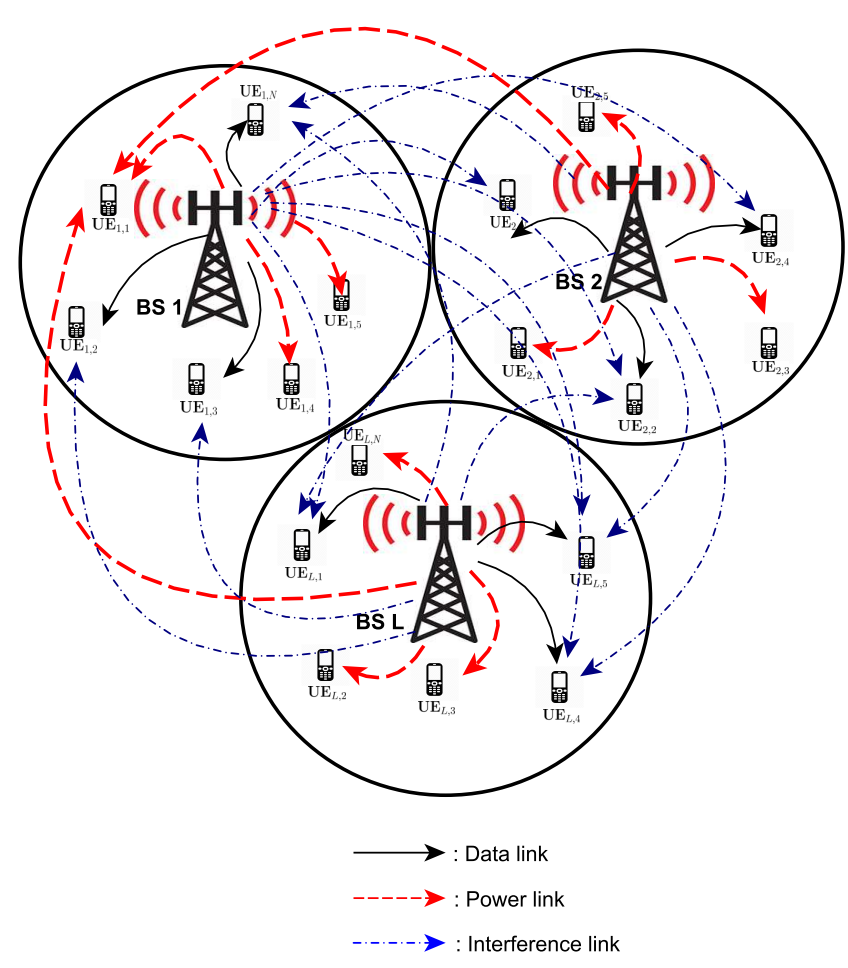

Fig. 1. System model of downlink multicell MU-MIMO SWIPT networks with IDRs and EHRs.

We consider the flat-fading channels in which the MIMO channel matrix from $\mathrm{BS}_{i}$ to $\mathrm{UE}_{\ell, k}$ is denoted by $\mathbf{H}_{\ell, k, i} \in \mathbb{C}^{N_{\ell, k} \times M_{i}}$. The channel coefficients accounts for both Rayleigh fading and path loss. We assume that the ideal CSI is available at the BSs and users $[4,5,22]$. As discussed in [26], [25], the multicell coordination can be divided into three categories: interference aware, joint processing and transmission, and coordinated beamforming. In this work, we focus on coordinated beamforming in which each BS can collect and pass the CSI associated its users to a central unit (which is connected to all the BSs via reliable high-speed channels) to design the precoding matrices [4]. We also remark that perfect CSI is difficult to obtain in practice and, then, the result in this paper provides the performance benchmark for imperfect CSI scenarios.

The received signal $\mathbf{y}_{\ell, \pi_{\ell}(k)} \in \mathbb{C}^{N_{\ell, \pi_{\ell}(k) \times 1}}$ at the selected $\mathrm{UE}_{\ell, \pi_{\ell}(\mathrm{k})}$ is given by

$$
\begin{aligned}
\mathbf{y}_{\ell, \pi_{\ell}(k)} & =\mathbf{H}_{\ell, \pi_{\ell}(k), \ell} \mathbf{V}_{\ell, \pi_{\ell}(k)} \mathbf{s}_{\ell, \pi_{\ell}(k)} \\
& +\sum_{s=1, s \neq k}^{S_{\ell}} \mathbf{H}_{\ell, \pi_{\ell}(s), \ell} \mathbf{V}_{\ell, \pi_{\ell}(s)} \mathbf{s}_{\ell, \pi_{\ell}(s)} \\
& +\sum_{i=1, i \neq \ell}^{L} \sum_{s=1}^{S_{i}} \mathbf{H}_{\ell, \pi_{\ell}(k), i} \mathbf{V}_{i, \pi_{i}(s)} \mathbf{s}_{i, \pi_{i}(s)}+\mathbf{n}_{\ell, \pi_{\ell}(k)}
\end{aligned}
$$

where $\mathbf{n}_{\ell, k} \sim \mathcal{C N}\left(0, \sigma_{\ell, k}^{2} \mathbf{I}\right)$ is additive white Gaussian noise at $\mathrm{UE}_{\ell, k}$. It is assumed that noise is independent with the transmitted signals. By treating interference as noise, the achievable rate of $\mathrm{UE}_{\ell, \pi_{\ell}(\mathrm{k})}$ from (1) is given by

$$
\begin{aligned}
& \mathcal{R}_{\ell, \pi_{\ell}(k)}(\mathbf{V})=\log \mid \mathbf{I}_{N_{\ell, \pi_{\ell}(k)}} \\
& +\mathbf{H}_{\ell, \pi_{\ell}(k), \ell} \mathbf{V}_{\ell, \pi_{\ell}(k)} \mathbf{V}_{\ell, \pi_{\ell}(k)}^{\mathrm{H}} \mathbf{H}_{\ell, \pi_{\ell}(k), \ell}^{\mathrm{H}} \mathbf{J}_{\ell, \pi_{\ell}(k)}^{-1} \mid
\end{aligned}
$$

where

$$
\begin{aligned}
\mathbf{J}_{\ell, \pi_{\ell}(k)}= & \sum_{(i, s) \neq(\ell, k)} \mathbf{H}_{\ell, \pi_{\ell}(k), i} \mathbf{V}_{i, \pi_{i}(s)} \mathbf{V}_{i, \pi_{i}(s)}^{\mathrm{H}} \mathbf{H}_{\ell, \pi_{\ell}(k), i}^{\mathrm{H}} \\
& +\sigma_{\ell, \pi_{\ell}(k)}^{2} \mathbf{I}_{d_{\ell, \pi_{\ell}(k)}}
\end{aligned}
$$

is the covariance matrix of interference-plus-noise at the selected user $\pi_{\ell}(k)$. Then, the SR of the network is

$$
\mathcal{R}_{\text {sum }}(\mathbf{V})=\sum_{\ell=1}^{L} \sum_{s=1}^{S_{\ell}} \mathcal{R}_{\ell, \pi_{\ell}(s)}(\mathbf{V}) .
$$

Inspired by the idea of [18], while the selected users receive information data from its associated BS, the unselected users can harvest power to recharge their batteries for the lifetime extension. The received signal $\mathbf{y}_{\ell, \phi_{\ell}(u)} \in \mathbb{C}^{N_{\ell, \phi_{\ell}(u)} \times 1}$ at the unselected user $\phi_{\ell}(u)$ in cell $\ell$ is given by

$$
\mathbf{y}_{\ell, \phi_{\ell}(u)}=\sum_{i=1}^{L} \sum_{s=1}^{S_{i}} \mathbf{H}_{\ell, \phi_{\ell}(u), i} \mathbf{V}_{i, \pi_{i}(s)} \mathbf{s}_{i, \pi_{i}(s)}+n_{\ell, \phi_{\ell}(u)} .
$$

As far as $\mathrm{EH}$ is concerned, the harvested energy is proportional to the received power at the baseband signals [12], [18]. Therefore, from (5), by ignoring noise power the harvested power at $\mathrm{UE}_{\ell, \phi_{\ell}(u)}$ can be defined by

$$
\begin{array}{r}
Q_{\ell, \phi_{\ell}(u)}(\mathbf{V})=\zeta_{\ell, \phi_{\ell}(u)} \sum_{i=1}^{L} \sum_{s=1}^{S_{i}}\left\langle\mathbf{H}_{\ell, \phi_{\ell}(u), i} \mathbf{V}_{i, \pi_{i}(s)}\right. \\
\left.\mathbf{V}_{i, \pi_{i}(s)}^{\mathrm{H}} \mathbf{H}_{\ell, \phi_{\ell}(u), i}^{\mathrm{H}}\right\rangle
\end{array}
$$

where $\zeta_{\ell, \phi_{\ell}(u)}$ accounts for the energy conversion efficiency which represents the loss in converting the harvested energy to electrical energy at the energy transducers of $\mathrm{UE}_{\ell, \phi_{\ell}(u)}$. Then, the total harvested energy in the multicell network is computed by

$$
Q_{\text {sum }}(\mathbf{V})=\sum_{\ell=1}^{L} \sum_{u=1}^{K_{\ell}-S_{\ell}} Q_{\ell, \phi_{\ell}(u)}(\mathbf{V}) .
$$

This paper aims at designing the precoders to simultaneously maximize the overall network data rate and harvested energy. Notice that to maximize the harvested energy at the EHRs, the power of the received signal at EHRs must increase and, then, interference to the IDR can increase which makes the degradation of the achievable SR. This means that the SR and SHE maximization are conflicting objectives. To investigate the trade-off between two objectives, we formulate the precoding design problem as multi-objective optimization. It should be noted that multi-objective functions of SR and SHE maximization can be transformed into a single-objective optimization problem by using weighted sum methods [20], [21]. Thus, our design problem is formulated as an optimization problem in which the objective is to maximize the weighted sum of the SR and the SHE subject to the transmit power constraints at each BS. The design of 
interest is mathematically formulated as

$$
\begin{array}{ll}
\max _{\mathbf{V}} & \alpha \frac{\mathcal{R}_{\text {sum }}(\mathbf{V})}{\mathcal{R}_{0}}+(1-\alpha) \frac{Q_{\text {sum }}(\mathbf{V})}{Q_{0}} \\
\text { s.t. } & \sum_{k=1}^{S_{\ell}}\left\langle\mathbf{V}_{\ell, \pi_{\ell}(k)} \mathbf{V}_{\ell, \pi_{\ell}(k)}^{\mathrm{H}}\right\rangle \leq P_{t_{\ell}}, \ell \in \mathcal{L}
\end{array}
$$

where the factors $\mathcal{R}_{0}, Q_{0}$ are used to normalize the SR and SHE into the same range. $P_{t_{\ell}}$ is a transmit power budget at $\mathrm{BS}_{\ell} .0 \leq \alpha \leq 1$ is the trade-off parameter which controls the priority between the SR and SHE in the objective function.

\section{Proposed Algorithm Derivation}

The objective function in (8a) is nonconcave and, thus, it renders mathematical difficulties to find the optimal precoders. In this section, we derive an iterative algorithm to solve problem (8).

\subsection{User Selection Strategy}

With regard to the user selection strategies, there are two conventional performance metrics, namely location-based selection and channel-gain-based selection [25]. In the former scheme, the BSs select users which have the smallest distance to the BSs while in the latter scheme the BSs select the users which have the highest channel strengths. The channel-gain-based selection schemes can provide better SR performance than the location-based selection. More recently, the authors in [26] proposed a user selection strategy for multicell systems based on the two linear precoding schemes namely, distributed zero-forcing and distributed virtual signal-to-interference-plus-noise ratio. Although the user selection introduced in [26] can provide superior sumrate performance, the selected users are relied on the structures of the precoding techniques. In this paper, we aim at designing the precoders to optimize the multi-objectives of the sum-rate and harvested energy and, thus, we decouple the user selection from the precoding designs. Therefore, we adopt the channel-gain-based selection scheme as the scheduling metric [4], [25]. The scheduling metric for user selection is defined as

$$
M_{\ell, k}=\left\langle\mathbf{H}_{\ell, k, \ell} \mathbf{H}_{\ell, k, \ell}^{\mathrm{H}}\right\rangle .
$$

In each transmission time slot, $\mathrm{BS}_{\ell}$ selects a set of $S_{\ell}$ users based on $S_{\ell}$ largest values of $M_{k, \ell}$ from all users in its cell.

\subsection{Optimization Algorithm Derivation}

Given the selected users, the design objective is to simultaneously maximize the SR and SHE in (8). It can be seen that neither SR nor SHE is a concave function and, thus, problem (8) is not a convex optimization problem. Therefore, it is mathematically challenging to obtain the optimal solutions to problem (8). To make problem (8) amenable, we exploit the connection between the MSE minimization and achievable data rate [23].

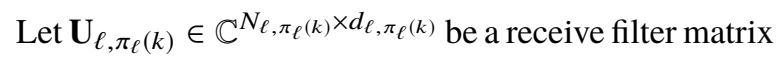
which is applied to decode the desired signals at $\mathrm{UE}_{\ell, \pi_{\ell}(\mathrm{k})}$ to yield

$$
\tilde{s}_{\ell, \pi_{\ell}(k)}=\mathbf{U}_{\ell, \pi_{\ell}(k)}^{\mathrm{H}} \mathbf{y}_{\ell, \pi_{\ell}(k)} .
$$

The MSE matrix for $\mathrm{UE}_{\ell, \pi_{\ell}(\mathrm{k})}$ is given by

$$
\begin{aligned}
& \mathbf{E}_{\ell, \pi_{\ell}(k)}=\mathbb{E}\left[\left(\tilde{s}_{\ell, \pi_{\ell}(k)}-s_{\ell, \pi_{\ell}(k)}\right)\left(\tilde{s}_{\ell, \pi_{\ell}(k)}-s_{\ell, \pi_{\ell}(k)}\right)^{\mathrm{H}}\right] \\
& =\sigma_{\ell, \pi_{\ell}(k)}^{2} \mathbf{U}_{\ell, \pi_{\ell}(k)}^{\mathrm{H}} \mathbf{U}_{\ell, \pi_{\ell}(k)}+\mathbf{I}+\mathbf{U}_{\ell, \pi_{\ell}(k)}^{\mathrm{H}} \times \\
& \left(\sum_{i=1}^{L} \sum_{s=1}^{S_{i}} \mathbf{H}_{\ell, \pi_{\ell}(k), i} \mathbf{V}_{i, \pi_{i}(s)} \mathbf{V}_{i, \pi_{i}(s)}^{\mathrm{H}} \mathbf{H}_{\ell, \pi_{\ell}(k), i}^{\mathrm{H}}\right) \mathbf{U}_{\ell, \pi_{\ell}(k)} \\
& -\mathbf{U}_{\ell, \pi_{\ell}(k)}^{\mathrm{H}} \mathbf{H}_{\ell, \pi_{\ell}(k), \ell} \mathbf{V}_{\ell, \pi_{\ell}(k)}-\mathbf{V}_{\ell, \pi_{\ell}(k)}^{\mathrm{H}} \mathbf{H}_{\ell, \pi_{\ell}(k), \ell}^{\mathrm{H}} \mathbf{U}_{\ell, \pi_{\ell}(k)} .
\end{aligned}
$$

Then, the minimization of MSE can be obtained at the optimal receive matrix $\mathbf{U}_{\ell, \pi_{\ell}(k)}$ given by

$$
\begin{aligned}
\mathbf{U}_{\ell, \pi_{\ell}(k)}^{\mathrm{opt}}= & \left(\sum_{i=1}^{L} \sum_{s=1}^{S} \mathbf{H}_{\ell, \pi_{\ell}(k), i} \mathbf{V}_{i, \pi_{i}(s)} \mathbf{V}_{i, \pi_{i}(s)}^{\mathrm{H}} \mathbf{H}_{\ell, \pi_{\ell}(k), i}^{\mathrm{H}}\right. \\
& \left.+\sigma^{2} \mathbf{I}_{N_{\ell, \pi_{\ell}(k)}}\right)^{-1} \mathbf{H}_{\ell, \pi_{\ell}(k), \ell} \mathbf{V}_{\ell, \pi_{\ell}(k) .}
\end{aligned}
$$

The derivations of (11) and (12) are provided in Appendix A. Substitution of $\mathbf{U}_{\ell, \pi_{\ell}(k)}^{\mathrm{opt}}$ into MSE matrix (11) yields

$$
\mathbf{E}_{\ell, \pi_{\ell}(k)}^{\mathrm{mmse}}=\mathbf{I}_{N_{\ell, \pi_{\ell}(k)}}-\mathbf{V}_{\ell, \pi_{\ell}(k)}^{\mathrm{H}} \mathbf{H}_{\ell, \pi_{\ell}(k), \ell}^{\mathrm{H}} \mathbf{U}_{\ell, \pi_{\ell}(k)}^{\mathrm{opt}},
$$

which can be equivalently rewritten as

$$
\begin{aligned}
& \mathbf{E}_{\ell, \pi_{\ell}(k)}^{\mathrm{mmse}}=\left(\mathbf{I}_{N_{\ell, \pi_{\ell}(k)}}\right. \\
& \left.+\mathbf{H}_{\ell, \pi_{\ell}(k), \ell} \mathbf{V}_{\ell, \pi_{\ell}(k)} \mathbf{V}_{\ell, \pi_{\ell}(k)}^{\mathrm{H}} \mathbf{H}_{\ell, \pi_{\ell}(k), \ell}^{\mathrm{H}} \mathbf{J}_{\ell, \pi_{\ell}(k)}^{-1}\right)^{-1} .
\end{aligned}
$$

Comparing (14) and (2), the relationship between MSE and $\mathrm{SR}$ of $\mathrm{UE}_{\ell, \pi_{\ell}(\mathrm{k})}$ can be established as

$$
\mathcal{R}_{\ell, \pi_{\ell}(k)}(\mathbf{V})=\log \left|\left(\mathbf{E}_{\ell, \pi_{\ell}(k)}^{\mathrm{mmse}}\right)^{-1}\right|
$$

Next, to tackle the difficulty associated with nonconcavity of the achievable rate function, we use the following inequality [23]

$$
\mathcal{R}_{\ell, \pi_{\ell}(s)}(\boldsymbol{V}) \geq f_{\ell, \pi_{\ell}(s)}(\mathbf{V}, \mathbf{U}, \mathbf{W})
$$

and, thus,

$$
\begin{aligned}
& \text { and, thus, } \\
& \mathcal{R}_{\text {sum }}(\mathbf{V}) \geq \hat{\mathcal{R}}_{\text {sum }}(\mathbf{V}, \mathbf{U}, \mathbf{W})=\sum_{\ell=1}^{L} \sum_{s=1}^{S_{\ell}} f_{\ell, \pi_{\ell}(s)}(\mathbf{V}, \mathbf{U}, \mathbf{W})
\end{aligned}
$$

where

$$
\begin{aligned}
f_{\ell, \pi_{\ell}(k)}(\mathbf{V}, \mathbf{U}, \mathbf{W}) & \triangleq \log \left|\mathbf{W}_{\ell, \pi_{\ell}(k)}\right| \\
& -\left\langle\mathbf{W}_{\ell, \pi_{\ell}(k)} \mathbf{E}_{\ell, \pi_{\ell}(k)}\right\rangle+d_{\ell, \pi_{\ell}(k)}
\end{aligned}
$$

and $\mathbf{W}_{\ell, \pi_{\ell}(k)} \in \mathbb{C}^{d_{\ell, \pi_{\ell}(k)}} \times d_{\ell, \pi_{\ell}(k)}$ is an auxiliary positive definite matrix variable. We have defined $\mathbf{U}=\left\{\mathbf{U}_{\ell, k}\right\}_{\ell \in \mathcal{L}, k \in \mathcal{K}_{\ell}}$ and $\mathbf{W}=\left\{\mathbf{W}_{\ell, k}\right\}_{\ell \in \mathcal{L}, k \in \mathcal{K}_{\ell}}$ for notation simplicity. It can be shown that the equality of (16) holds at $\mathbf{U}_{\ell, \pi_{\ell}(k)}^{\mathrm{opt}}$ given in (12) and

$$
\mathbf{W}_{\ell, \pi_{\ell}(k)}^{\mathrm{opt}}=\left(\mathbf{E}_{\ell, \pi_{\ell}(k)}^{\mathrm{mmse}}\right)^{-1} .
$$


Next, to handle the non-concavity of the harvested energy function, we exploit its convexity. Given feasible matrices $\mathbf{V}_{i, \pi_{i}(k)}^{(n)}$, the convex quadratic function of the harvested energy is lower-bounded by its minorant as follows [24], [22]

$$
Q_{\ell, \phi_{\ell}(u)}(\mathbf{V}) \geq Q_{\ell, \phi_{\ell}(u)}^{(n)}(\mathbf{V})
$$

where

$$
\begin{gathered}
Q_{\ell, \phi_{\ell}(u)}^{(n)}(\mathbf{V})=\zeta_{\ell, \phi_{\ell}(u)} \sum_{i=1}^{L} \sum_{s=1}^{S_{i}}\left\langle\mathbf{H}_{\ell, \phi_{\ell}(u), i} \mathbf{V}_{i, \pi_{i}(s)}^{(n)}\right. \\
\left.\mathbf{V}_{i, \pi_{i}(s)}^{(n) \mathrm{H}} \mathbf{H}_{\ell, \phi_{\ell}(u), i}^{\mathrm{H}}\right\rangle+ \\
\zeta_{\ell, \phi_{\ell}(u)} \sum_{i=1}^{L} \sum_{s=1}^{S_{i}}\left\langle\mathbf{V}_{i, \pi_{i}(s)}^{(n) \mathrm{H}} \mathbf{H}_{\ell, \phi_{\ell}(u), i}^{\mathrm{H}} \mathbf{H}_{\ell, \phi_{\ell}(u), i}\right. \\
\left.\zeta_{\ell, \phi_{\ell}(u)} \sum_{i=1}^{L} \sum_{s=1}^{S_{i}}\left\langle\left(\mathbf{V}_{i, \pi_{i}(s)}-\mathbf{V}_{i, \pi_{i}(s)}^{(n)}\right)^{\mathrm{H}} \mathbf{H}_{\ell, \phi_{\ell}(u), i}^{\mathrm{H}}-\mathbf{V}_{i, \pi_{i}(s)}^{(n)}\right)\right\rangle+ \\
\left.\mathbf{H}_{\ell, \phi_{\ell}(u), i} \mathbf{V}_{i, \pi_{i}(s)}^{(n)}\right\rangle .
\end{gathered}
$$

Note that $Q_{\ell, \phi_{\ell}(u)}\left(\mathbf{V}^{(n)}\right)=Q_{\ell, \phi_{\ell}(u)}^{(n)}\left(\mathbf{V}^{(n)}\right)$. Thus, the lower bound of the SHE at iteration $n$ is

$$
\boldsymbol{Q}_{\text {sum }}(\mathbf{V}) \geq \widehat{Q}_{\text {sum }}^{(n)}(\mathbf{V})=\sum_{\ell=1}^{L} \sum_{u=1}^{N_{\ell}-S_{\ell}} Q_{\ell, \phi_{\ell}(u)}^{(n)}(\mathbf{V}) .
$$

Thus, we can solve the nonconvex optimization problem (8) by iteratively solving the following optimization problem

$$
\begin{array}{ll}
\max _{\{\mathbf{V}, \mathbf{U}, \mathbf{W}\}} & \alpha \frac{\widehat{\mathcal{R}}_{\text {sum }}(\{\mathbf{V}, \mathbf{U}, \mathbf{W}\})}{\mathcal{R}_{0}}+(1-\alpha) \frac{\widehat{Q}_{\text {sum }}^{(n)}(\mathbf{V})}{Q_{0}} \\
\text { s.t. } & (8 \mathrm{~b})
\end{array}
$$

It can be observed that the objective function in (23) is not concave with respect to three set of variables $\{\mathbf{V}, \mathbf{U}, \mathbf{W}\}$, but it is concave over each set of variables when the other two sets are fixed. Thus, an AO approach is applicable to iteratively solve (23). More specifically, at each iteration, we alternatively find the three blocks of variables as follows:

- Fix $\{\mathbf{V}, \mathbf{W}\}$ and update $\mathbf{U}$. Finding $\mathbf{U}$ to maximize the object function in (23) is equivalent to minimize the MSE and, thus, $\mathbf{U}$ can be found from (12).

- Fix $\{\mathbf{V}, \mathbf{U}\}$ and update $\mathbf{W}$. It can be shown that problem (23) is a convex optimization with variable $\mathbf{W}$. Thus by setting the first derivation the object function in (23) with respect to $\mathbf{W}$ to zero, we can obtain $\mathbf{W}$ from (19).

- Fixed $\{\mathbf{U}, \mathbf{W}\}$ and update $\mathbf{V}$. It can be observed that the objective function in (23) is a concave function with respect to variable $\mathbf{V}$. Thus, update $\mathbf{V}$ by solving the problem (23) using convex optimization solvers, e.g., CVX [27].

To further clarify the proposed algorithm, we summarize the detailed procedure to solve problem (8) in Algorithm 1.

\subsection{Convergence and Computational Complexity Analysis}

Let us define the objective functions in (8a) and (23a) as $f(\mathbf{V})$ and $\hat{f}(\mathbf{V}, \mathbf{W}, \mathbf{U})$, respectively. By denoting $\left(\mathbf{V}^{(n+1)}, \mathbf{W}^{(n+1)}, \mathbf{U}^{(n+1)}\right)$ is the solution after iteration $(n+1)$ in Algorithm 1, we have

$$
\begin{aligned}
f\left(\mathbf{V}^{(n+1)}\right) & \geq \hat{f}\left(\mathbf{V}^{(n+1)}, \mathbf{W}^{(n+1)}, \mathbf{U}^{(n+1)}\right) \\
& \geq \hat{f}\left(\mathbf{V}^{(n)}, \mathbf{W}^{(n)}, \mathbf{U}^{(n)}\right) \\
& =f\left(\mathbf{V}^{(n)}\right)
\end{aligned}
$$

where inequality (24a) holds since $\hat{f}(\mathbf{V}, \mathbf{W}, \mathbf{U})$ is a lower bound of $f(\mathbf{V})$, inequality (24b) holds because $\left(\mathbf{V}^{(n+1)}, \mathbf{W}^{(n+1)}, \mathbf{U}^{(n+1)}\right)$ is the optimal solution to problem (23) at iteration $(n+1)$, and $(24 c)$ is true due to the fact that equalities (17) and (22) holds at $\left(\mathbf{V}^{(n)}, \mathbf{W}^{(n)}, \mathbf{U}^{(n)}\right)$. Thus, the objective function $f(\mathbf{V})$ of problem (8) is not decreasing over iterations. In addition, with given transmitted power constraints, the objective function is upper bounded. Therefore, the convergence of Algorithm 1 is guaranteed.

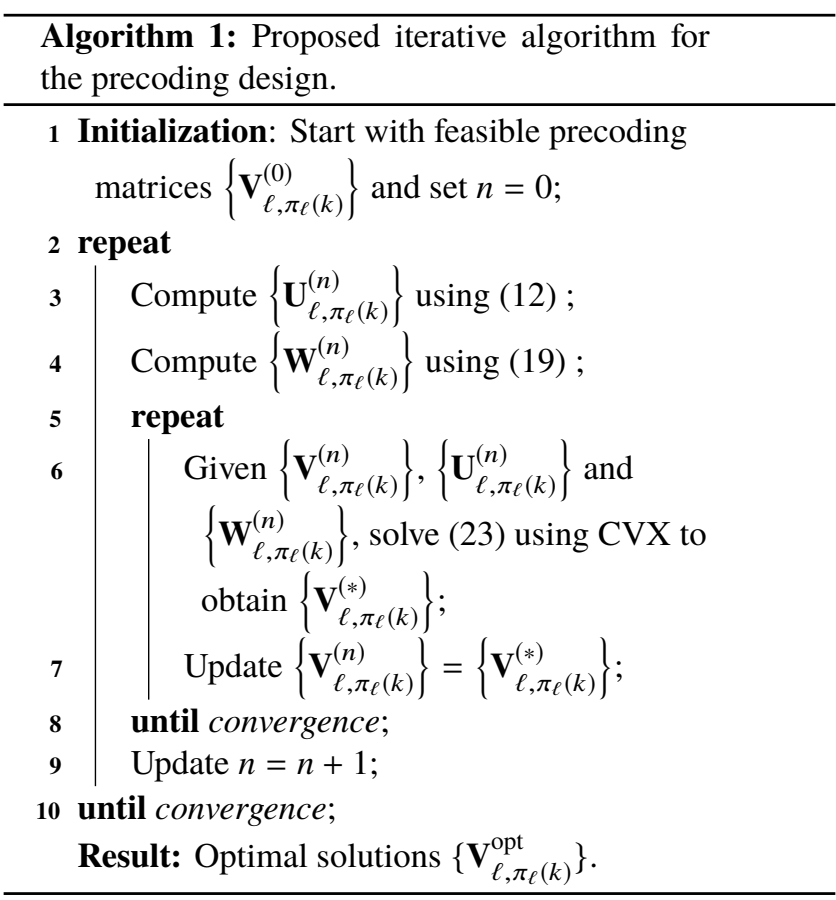

Now, we study the computational complexity of the proposed iterative algorithm. The major computational complexity of Algorithm 1 includes the complexity $O\left(\sum_{\ell=1}^{L} \sum_{k=1}^{S_{\ell}} N_{\ell, \pi_{\ell}(k)}^{3}\right)$ for calculating $\mathbf{U}, O\left(\sum_{\ell=1}^{L} \sum_{k=1}^{S_{\ell}} d_{\ell, \pi_{\ell}(k)}^{3}\right)$ for obtaining $\mathbf{W}$, and $O\left(N_{\max }\left(\sum_{\ell=1}^{L} \sum_{k=1}^{S_{\ell}} M_{\ell} d_{\ell, \pi_{\ell}(k)}\right)^{3}\right)$ solving the convex optimization (23) to obtain $\mathbf{V}$ where $N_{\text {max }}$ is an average iteration for the inner loop in Algorithm 1 to be converged [14], [23]. In numerical simu- 
lations, $N_{\max }$ is a few tens of iterations. Thus, the total computational complexity for each iteration is about $O\left(\sum_{\ell=1}^{L} \sum_{k=1}^{S_{\ell}}\left(N_{\ell, \pi_{\ell}(k)}^{3}+d_{\ell, \pi_{\ell}(k)}^{3}\right)+N_{\max }\left(\sum_{\ell=1}^{L} \sum_{k=1}^{S_{\ell}} M_{\ell} d_{\ell, \pi_{\ell}(k)}\right)^{3}\right)$ which reveals the low computational complexity of Algorithm 1.

\section{Simulation Results}

In this section, we evaluate the effectiveness of our iterative algorithm and investigate the trade-off between the SR and SHE performance via numerical simulation results. In simulations, the considered systems consist of three $40 \mathrm{~m}$ radius cells. All BSs transmit 2 data streams to each UE $\left(d_{\ell, k}=d=2\right)$. Each UE has 2 antennas $\left(N_{\ell, k}=N=2\right)$. The users are randomly placed in each cell. Assume that path loss at distance $\mathrm{r}(\mathrm{m})$ between a BS and an UE is given by $31.7+27.6 \log _{10}\left(\frac{r}{r_{0}}\right) \mathrm{dB}$ where $r_{0}=1$ (m) is a reference distance [19]. The noise power is set as $P_{N}=\sigma_{\ell, k}^{2}=-99 \mathrm{dBm}[19]$. For the sake of simplicity, the energy conversion efficiency $\zeta_{\ell, \phi_{\ell}(u)}=1$. The transmit power budget of all BSs are assumed to be equal $P_{t_{\ell}}=P_{t}$. We denote the system configuration of $K_{\ell}=K$ users in each cell in which there are $S_{\ell}=S$ IDRs and $K-S$ EHRs as $\{K, S, K-S\}$. All numerical simulations are conducted in MATLAB on Intel Core i7-6500U CPU $2.6 \mathrm{GHz}$ with RAM 16 GB. To obtain the optimal precoders, the convex optimization problems (23) are solved by the CVX package with the internal solver SDPT3 [27].

Example 1: This example investigates the convergence behaviors of iterative Algorithm 1 for the system model $\{4,2,2\}$ and each BS equipped with 4 antennas $\left(M_{\ell}=M=4\right)$ and $P_{t}=30 \mathrm{dBm}$. The normalization factors are set as $\mathcal{R}_{0}=70 \mathrm{bps} / \mathrm{Hz}$ and $Q_{0}=1.5 P_{N} \times 10^{7}$. Figure 2 depicts the evolution of the objective function $f\left(\mathbf{V}^{(n)}\right)$ over iterations for a given random channel realization. It can be observed that for different values of $\alpha$, the objective functions are not decreasing over iterations and the objective functions are converged within a less than 50 iterations. The similar convergence characteristics can be observed for the other system models considered below and, thus, we do not provide the simulation results for the other models.

Example 2: In this example, we investigate the tradeoffs between the SR and SHE for the multicell MU-MIMO SWIPT networks with various configuration settings. We carried out 100 Monte Carlo runs with random locations of users to obtain average results. Remark that for $\alpha=1$ the multi-objective optimization problem (8) reduces to the SR maximization and, thus, the SR is highest and the SHE is lowest. In contrast, the systems will offer the highest SHE and lowest SR for $\alpha=0$. Thus, in the following, to normalize the SR and SHE (8), $\mathcal{R}_{0}$ is set as the highest achievable SR of (8) at $\alpha=1$ and $Q_{0}$ is the highest SHE at $\alpha=0$.

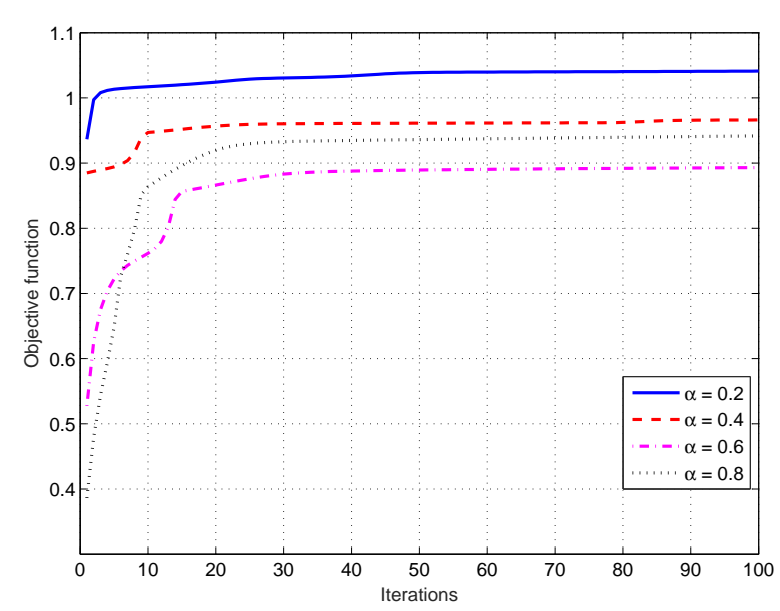

Fig. 2. Convergence behaviors of iterative Algorithm 1.

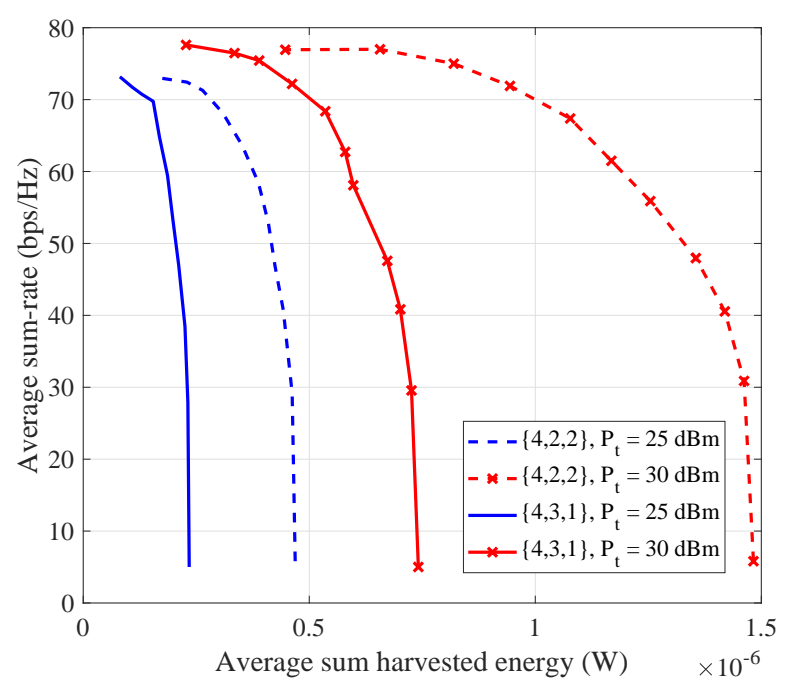

Fig. 3. The trade-off characteristics between the SR and SHE for $\{4,2,2\}$ and $\{4,3,1\}$ systems.

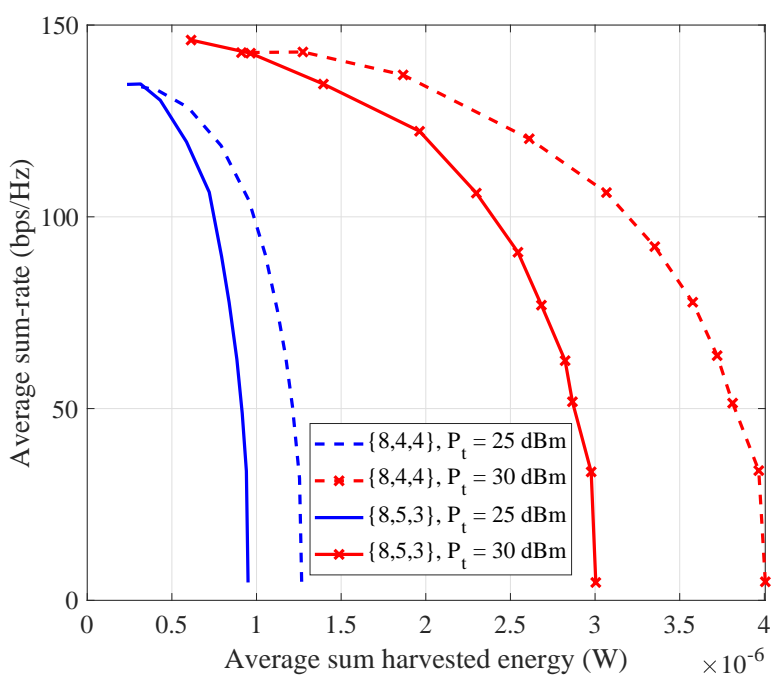

Fig. 4. The trade-off characteristics between the SR and SHE for $\{8,4,4\}$ and $\{8,5,3\}$ systems. 
We consider the system configurations of $\{4,2,2\}$ and $\{4,3,1\}$ in which each BS is equipped with 4 transmit antennas. Figure 3 illustrates the achievable trade-off curves between the SR and SHE which are obtained by varying the trade-off parameter $\alpha$. The SR from Fig. 3 is monotonically reduced when the SHE is increased. This indicates that the SR and SHE maximization objectives are conflicting. As can be seen from Fig. 3, the system achieves the highest SR at $\alpha=1$ where the achievable SHE is lowest. On the other hand, the system obtains the lowest SR when the SHE is highest at $\alpha=0$.

The similar results can be observed from Fig. 4 for the system configurations of $\{8,4,4\}$ and $\{8,5,3\}$ with each BS equipped with 8 antennas. Thus, the trade-off parameter $\alpha$ in problem (8) provides the flexibility to switch between the SE and harvested energy efficiency. Additionally, the SR and SHE region from Figs. 3 and 4 expands with the increase of $P_{t}$, this means, the amount of both SR and SHE become larger if the transmit power budget is higher. In addition, when the number of IDRs increases (i.e, the number of EHRs is reduced) the achievable SHE is significantly degraded while there is only a slight increase in the achievable SR (it can be observed in Figs. 3 and 4 at points $\alpha=1$ and $\alpha=0$ ). The reason is that the optimal precoder solutions tend to allocate more power to the user with the better channel-gain condition. Thus, the increase in the number of IDRs can lead to an insignificant increase in the achievable sum-rate while the reduction in the number of EHRs can significantly degrade the achievable SHE.

\section{Conclusion}

We have presented an efficient iterative algorithm for the precoding design to maximize simultaneously two performance metrics, namely SR and SHE, in multi-cell MIMO SWIPT systems under transmit power constraints at each BS. We have exploited the relationship between MSE minimization and achievable data rate to transform the SR function into concave one in each set of variables, and we have also derived the concave minorant of the SHE function. Then, we have developed an efficient iterative algorithm with low complexity and guaranteed convergence. The simulation results have verified the fast convergence of the proposed iterative algorithm. In addition, the simulation results have provided useful insights into the trade-offs between the SR and SHE for various transmit power levels and the different number of users.

\section{Acknowledgments}

This research is funded by Vietnam National Foundation for Science and Technology Development (NAFOSTED) under grant number 102.04-2017.308.

\section{References}

[1] GUPTA, A., JHA, R. K. A survey of 5G network: architecture and emerging technolgies. IEEE Access, 2015, vol. 3, p. 1206-1232. DOI: 10.1109/ACCESS.2015.2461602

[2] VU, T. T., KHA, H, H, DUONG, T. Q., et al. Particle swarm optimization for weighted sum rate maximization in MIMO broadcast channels. Wireless Personal COmmunications, 2017, vol. 96, no. 3, p. 2907-3921. DOI: $10.1007 / \mathrm{s} 11277-017-4357-2$

[3] TAM, H. H. M., TUAN, H. D., NGO, D. T. Successive convex quadratic programming for quality of service management in full-duplex MU-MIMO multicell networks. IEEE Transactions on Communications, 2016, vol. 64, no. 6, p. 2340-2353. DOI: 10.1109/TCOMM.2016.2550440

[4] KHA, H. H. Interference mitigation and sum rate optimization for MIMO downlink small cells. Radioengineering, 2016, vol. 25, no. 4, p. 721-729. DOI: 10.13164/re.2016.0721

[5] TANG, J., SO, D. K. C., ZHAO, N., et al. Energy efficiency optimization with SWIPT in MIMO broadcast channels for Internet of things. IEEE Internet of Things Journal, 2018, vol. 5, no. 4, p. 2605-2619. DOI: 10.1109/JIOT.2017.2785861

[6] KHA, H. H., TUNG, T. V., DO-HONG, T. Energy-efficient transceiver designs for multiuser MIMO cognitive radio networks via interference alignment. Telecommunication Systems, 2017, vol. 66, no. 3, p. 469-480. DOI: $10.1007 / \mathrm{s} 11235-017-0300-9$

[7] VARSHNEY, L. R. Transporting information and energy simultaneously. In Proceedings of the IEEE International Symposium on Information Theory. Toronto (Canada), 2008, p. 1612-1616. DOI: $10.1109 /$ ISIT.2008.4595260

[8] XU, J., LIU, L., ZHANG, R. Multiuser MISO beamforming for simultaneous wireless information and power transfer. IEEE Transactions on Signal Processing, 2014, vol. 62, no. 18, p. 4754-4758. 10.1109/TSP.2014.2340817

[9] ZHOU, X., ZHANG, R., HO, C. K. Wireless information and power transfer in multiuser OFDM systems. IEEE Transactions on Wireless Communications, 2014, vol. 13, no. 4, p. 2282-2294. DOI: 10.1109/TWC.2014.030514.131479

[10] KHANDAKER, M. R. A., WONG, K.-K. SWIPT in MISO multicasting systems. IEEE Wireless Communications Letters, 2014, vol. 3, no. 3, p. 277-280. DOI: 10.1109/WCL.2014.030514.140057

[11] SON, H., CLERCKX, B. Joint beamforming design for multi-user wireless information and power transfer. IEEE Transactions on Wireless Communications, 2014, vol. 13, no. 11, p. 6397-6409. DOI: 10.1109/TWC.2014.2349511

[12] ZHANG, R., HO, C. K. MIMO broadcasting for simultaneous wireless information and power transfer. IEEE Transactions on Wireless Communications, 2013, vol. 12, no. 5, p. 781-2001. DOI: 10.1109/TWC.2013.031813.120224

[13] NG, D. W. K., LO, E. S., SCHOBER, R. Multiobjective resource allocation for secure communication in cognitive radio networks with wireless information and power transfer. IEEE Transactions on Vehicular Technology, 2016, vol. 65, no. 6, p. 3166-3184. DOI: $10.1109 /$ TVT.2015.2436334

[14] ZONG, Z., FENG, H., YU, F. R., et al. Optimal transceiver design for SWIPT in K-user MIMO interference channel. IEEE Transactions on Wireless Communications, 2016, vol. 15, no. 1, p. 430-455. DOI: 10.1109/TWC.2015.2474857

[15] PARK, J., CLERCKX, B. Joint wireless information and energy transfer in a K-user MIMO interference channel. IEEE Transactions on Wireless Communications, 2014, vol. 13, no. 10, p. 5781-5796. DOI: 10.1109/TWC.2014.2341233 
[16] NASIR, A. A., TUAN, H. D., DUONG, T. Q., POOR, H. V. Secure and energy efficient beamforming for simultaneous information and energy transfer. IEEE Transactions on Wireless Communications, 2017, vol. 16, no. 11, p. 7523-7537. DOI: 10.1109/TWC.2017.2749568

[17] SHI, Q., XU, W., WU, J., SONG, E., WANG, Y. Secure beamforming for MIMO broadcasting with wireless information and power transfer. IEEE Transactions on Wireless Communications, 2015, vol. 14, no. 5, p. 2841-2853. DOI: 10.1109/TWC.2015.2395414

[18] REN, Y., LV, T., GAO, H., YANG, S. Wireless information and energy transfer in multi-cluster MIMO uplink networks through opportunistic interference alignment. IEEE Access, 2016, vol. 4, p. 3100-3111. DOI: 10.1109/ACCESS.2016.2580681

[19] BANG, I., KIM, S. M., SUNG, D. K. Adaptive multiuser scheduling for simultaneous wireless information and power transfer in a multicell environment. IEEE Transactions on Wireless Communications, 2017, vol. 16, no. 11, p. 7460-7474. DOI: 10.1109/TWC.2017.2748942

[20] RUBIO, J., PASCUAL-ISERTE, A., PALOMAR, D. P., et al. Joint optimization of power and data transfer in multiuser MIMO systems. IEEE Transactions on Signal Processing, 2017, vol. 65, no. 1, p. 212-227. DOI: 10.1109/TSP.2016.2614794

[21] AMIN, O., BEDEER, E., AHMED, M. H., et al. Energy efficiencyspectral efficiency trade-off: a multiobjective optimization approach. IEEE Transactions on Vehicular Technology, 2016, vol. 65, no. 4, p. 1975-1981. DOI: 10.1109/TVT.2015.2425934

[22] TAM, H.H.M., TUAN, H.D., et al. MIMO energy harvesting in full-duplex multi-user networks. IEEE Transactions on Wireless Communications, 2017, vol. 16, no. 5, p. 3282-3297. DOI: $10.1109 /$ TWC.2017.2679055

[23] SHI, Q., RAZAVIVAVN, M., LUO, Z., HE, C. An iteratively weighted MMSE approach to distributed sum-utility maximization for a MIMO interfering broadcast channel. IEEE Transaction on Signal Processing, 2011, p. 4331-4340. DOI: 10.1109/TSP.2011.2147784

[24] KHA, H.H., TUAN, H.D., NGUYEN, H. H. Fast global optimal power allocation in wireless networks by local D.C. programming. IEEE Transactions on Wireless Communications, 2012, vol. 11, no. 2, p. 510-515. DOI: 10.1109/TWC.2011.120911.110139

[25] NGUYEN, D. H. N, LE-NGOC, T. Joint beamforming design and base-station assignment in a coordinated multicell system. IET Communications, 2013, vol. 7, no. 10, p. 942-949. DOI: $10.1049 /$ iet-com.2012.0603

[26] CASTANEDA, E., SILVA, A., SAMANO-ROBLES, R. Distributed linear precoding and user selection in coordinated multicell systems. EEE Transactions on Vehicular Technology, 2015, vol. 65, no. 7, p. 4887-4899. DOI: 10.1109/TVT.2015.2455596

[27] GRANT, M., BOYD, S., YE, Y. CVX: Matlab software for disciplined convex programming, 2014. Available at: http://cvxr.com/cvx

\section{About the Authors...}

Tien Ngoc HA obtained the B.Eng. degree in Electronics and Telecommunications Engineering from Ho Chi Minh City University of Technology in 2018. He is currently a postgraduate student at the Faculty of Electrical and Electronics Engineering, Ho Chi Minh City University of Technology. His research interests are the areas of signal processing and wireless communication systems.
Ha Hoang KHA (corresponding author) received the B.Eng. and M.Eng. degrees from Ho Chi Minh City University of Technology, in 2000 and 2003, respectively, and the Ph.D. degree from the University of New South Wales, Sydney, Australia, in 2009, all in Electrical Engineering and Telecommunications. From 2000 to 2004, he was a research and teaching assistant with the Department of Electrical and Electronics Engineering, Ho Chi Minh City University of Technology. He was a visiting research fellow at the School of Electrical Engineering and Telecommunications, the University of New South Wales, Australia, from 2009 to 2011. He was a postdoctoral research fellow at the Faculty of Engineering and Information Technology, University of Technology Sydney, Australia from 2011 to 2013. He is currently a lecturer at the Faculty of Electrical and Electronics Engineering, Ho Chi Minh City University of Technology, Vietnam. His research interests are in digital signal processing and wireless communications, with a recent emphasis on convex optimization techniques in signal processing for wireless communications.

\section{Appendix A: Derivations of Equations (11) and (12)}

The mean-squared-error (MSE) matrix for $\mathrm{UE}_{\ell, \pi_{\ell}(\mathrm{k})}$ is given by

$$
\begin{gathered}
\mathbf{E}_{\ell, \pi_{\ell}(k)}=\mathbb{E}\left[\left(\tilde{s}_{\ell, \pi_{\ell}(k)}-\mathbf{s}_{\ell, \pi_{\ell}(k)}\right)\left(\tilde{s}_{\ell, \pi_{\ell}(k)}-\mathbf{s}_{\ell, \pi_{\ell}(k)}\right)^{\mathrm{H}}\right] \\
=\mathbb{E}\left[\left(\mathbf{U}_{\ell, \pi_{\ell}(k)}^{\mathrm{H}} \mathbf{y}_{\ell, \pi_{\ell}(k)}-\mathbf{s}_{\ell, \pi_{\ell}(k)}\right)\right. \\
\left.\left(\mathbf{U}_{\ell, \pi_{\ell}(k)}^{\mathrm{H}} \mathbf{y}_{\ell, \pi_{\ell}(k)}-\mathbf{s}_{\ell, \pi_{\ell}(k)}\right)^{\mathrm{H}}\right]
\end{gathered}
$$

which can be rewritten as in (A2) (at the top of next page).

With the independence assumptions of the transmitted signals and noise, we have $\mathbb{E}\left[\mathbf{s}_{\ell, k} \mathbf{s}_{i, j}^{\mathrm{H}}\right]=0$ for $(\ell, k) \neq(i, j)$, $\mathbb{E}\left[\mathbf{s}_{\ell, k} \mathbf{s}_{\ell, k}^{\mathrm{H}}\right]=\mathbf{I}, \mathbb{E}\left[\mathbf{s}_{\ell, k} \mathbf{n}_{i, j}^{\mathrm{H}}\right]=0, \mathbb{E}\left[\mathbf{n}_{\ell, k} \mathbf{n}_{i, j}^{\mathrm{H}}\right]=0$ for $(\ell, k) \neq(i, j)$ and $\mathbb{E}\left[\mathbf{n}_{\ell, k} \mathbf{n}_{\ell, k}^{\mathrm{H}}\right]=\sigma_{\ell, k}^{2} \mathbf{I}$. Then, the MSE matrix in (A2) becomes

$$
\begin{aligned}
& \mathbf{E}_{\ell, \pi_{\ell}(k)}=\mathbf{U}_{\ell, \pi_{\ell}(k)}^{\mathrm{H}} \mathbf{H}_{\ell, \pi_{\ell}(k), \ell} \mathbf{V}_{\ell, \pi_{\ell}(k)} \mathbf{V}_{\ell, \pi_{\ell}(k)}^{\mathrm{H}} \mathbf{H}_{\ell, \pi_{\ell}(k), \ell}^{\mathrm{H}} \mathbf{U}_{\ell, \pi_{\ell}(k)} \\
& +\mathbf{U}_{\ell, \pi_{\ell}(k)}^{\mathrm{H}} \sum_{(\ell, k) \neq(s, i)} \mathbf{H}_{\ell, \pi_{\ell}(k), i} \mathbf{V}_{i, \pi_{i}(s)} \mathbf{V}_{i, \pi_{i}(s)}^{\mathrm{H}} \mathbf{H}_{\ell, \pi_{\ell}(k), i}^{\mathrm{H}} \mathbf{U}_{\ell, \pi_{\ell}(k)} \\
& -\mathbf{U}_{\ell, \pi_{\ell}(k)}^{\mathrm{H}} \mathbf{H}_{\ell, \pi_{\ell}(k), \ell} \mathbf{V}_{\ell, \pi_{\ell}(k)}-\mathbf{V}_{\ell, \pi_{\ell}(k)}^{\mathrm{H}} \mathbf{H}_{\ell, \pi_{\ell}(k), \ell}^{\mathrm{H}} \mathbf{U}_{\ell, \pi_{\ell}(k)} \\
& +\sigma_{\ell, \pi_{\ell}(k)}^{2} \mathbf{U}_{\ell, \pi_{\ell}(k)}^{\mathrm{H}} \mathbf{U}_{\ell, \pi_{\ell}(k)}+\mathbf{I}_{\ell, \pi_{\ell}(k)}
\end{aligned}
$$

which can be rewritten as (11). 


$$
\begin{aligned}
\mathbf{E}_{\ell, \pi_{\ell}(k)} & =\mathbb{E}\left[\left(\mathbf{U}_{\ell, \pi_{\ell}(k)}^{\mathrm{H}}\left(\mathbf{H}_{\ell, \pi_{\ell}(k), \ell} \mathbf{V}_{\ell, \pi_{\ell}(k)} \mathbf{s}_{\ell, \pi_{\ell}(k)}+\sum_{(\ell, k) \neq(s, i)} \mathbf{H}_{\ell, \pi_{\ell}(k), i} \mathbf{V}_{i, \pi_{i}(s)} \mathbf{s}_{i, \pi_{i}(s)}+n_{\ell, \pi_{\ell}(k)}\right)-\mathbf{s}_{\ell, \pi_{\ell}(k)}\right)\right. \\
& \left.\times\left(\mathbf{U}_{\ell, \pi_{\ell}(k)}^{\mathrm{H}}\left(\mathbf{H}_{\ell, \pi_{\ell}(k), \ell} \mathbf{V}_{\ell, \pi_{\ell}(k)} \mathbf{s}_{\ell, \pi_{\ell}(k)}+\sum_{(\ell, k) \neq(s, i)} \mathbf{H}_{\ell, \pi_{\ell}(k), i} \mathbf{V}_{i, \pi_{i}(s)} \mathbf{s}_{i, \pi_{i}(s)}+n_{\ell, \pi_{\ell}(k)}\right)-\mathbf{s}_{\ell, \pi_{\ell}(k)}\right)^{\mathrm{H}}\right]
\end{aligned}
$$

The MSE for $\mathrm{UE}_{\ell, \pi_{\ell}(\mathrm{k})}$ is defined as $\mathrm{MSE}_{\ell, \pi_{\ell}(k)}=$ $\left\langle\mathbf{E}_{\ell, \pi_{\ell}(k)}\right\rangle$. An optimal receive matrix $\mathbf{U}_{\ell, \pi_{\ell}(k)}$ which minimizes the MSE can be obtained by solving $\partial\left\langle\mathbf{E}_{\ell, \pi_{\ell}(k)}\right\rangle / \partial \mathbf{U}_{\ell, \pi_{\ell}(k)}^{*}=\mathbf{0}$. From (11), we have

$$
\begin{aligned}
& \frac{\partial\left\langle\mathbf{E}_{\ell, \pi_{\ell}(k)}\right\rangle}{\partial \mathbf{U}_{\ell, \pi_{\ell}(k)}^{*}}=\sum_{i=1}^{L} \sum_{s=1}^{S_{i}} \mathbf{H}_{\ell, \pi_{\ell}(k), i} \mathbf{V}_{i, \pi_{i}(s)} \mathbf{V}_{i, \pi_{i}(s)}^{\mathrm{H}} \mathbf{H}_{\ell, \pi_{\ell}(k), i}^{\mathrm{H}} \mathbf{U}_{\ell, \pi_{\ell}(k)} \\
& -\mathbf{H}_{\ell, \pi_{\ell}(k), \ell} \mathbf{V}_{\ell, \pi_{\ell}(k)}+\sigma_{\ell, \pi_{\ell}(k)}^{2} \mathbf{U}_{\ell, \pi_{\ell}(k)}=\mathbf{0}
\end{aligned}
$$

which results in (12). 